\title{
Omental synovial sarcoma mimicking an ovarian malignancy: A case report
}

\author{
NAOYUKI IWAHASHI ${ }^{1,2}$, YOKO DEGUCHI ${ }^{1,2}$, YUKO HORIUCHI ${ }^{1,2}$, KAZUHIKO INO $^{2}$ and KENICHI FURUKAWA $^{1}$ \\ ${ }^{1}$ Department of Obstetrics and Gynecology, Hashimoto Municipal Hospital, Hashimoto, Wakayama 648-0005; \\ ${ }^{2}$ Department of Obstetrics and Gynecology, Wakayama Medical University, Wakayama 641-0012, Japan
}

Received November 14, 2016; Accepted March 17, 2017

DOI: $10.3892 /$ mco.2017.1213

\begin{abstract}
Synovial sarcoma is clinically rare, and cases of synovial sarcoma arising in the omentum are particularly rare. Only 3 cases have been reported in the literature to dtae, and they were associated with a poor prognosis. We herein report a rare case of aggressive primary omental synovial sarcoma presenting as an ovarian malignancy. A 53-year-old multigravida woman was referred to our hospital due to progressive abdominal distension. Magnetic resonance imaging revealed a large heterogeneous mass with an irregular component occupying the lower abdominal cavity, with an intact uterus. Intraoperative examination revealed a solid mass arising from the lower omentum. The diagnosis of omental synovial sarcoma was established based on postoperative pathological and immunohistochemical examination of the tumor. The patient underwent multiple surgical resection procedures due to the development of metastases in the liver, lungs and abdominal cavity and received adjuvant chemotherapy including doxorubicin-ifosfamide, pazopanib and trabectedin. Such rare neoplasms may be difficult to diagnose preoperatively based on radiological examinations alone. Thus, gynecologists should be aware of the possibility of omental synovial sarcoma, and combined surgical and chemotherapeutic interventions are required to control such aggressive tumors.
\end{abstract}

\section{Introduction}

Synovial sarcoma is a clinically rare neoplasm that accounts for $\sim 10 \%$ of all malignant soft tissue tumors. Synovial sarcoma most commonly arises in the soft tissues of the extremities in young and middle-aged adults (1). This tumor may also arise in other parts of the body, but primary omental synovial sarcoma is extremely rare. To the best of our knowledge, only 3 cases of primary omental synovial sarcoma ( 2 cases in women and

Correspondence to: Dr Naoyuki Iwahashi, Department of Obstetrics and Gynecology, Wakayama Medical University, 811-1 Kimiidera, Wakayama 641-0012, Japan

E-mail: naoyuki@wakayama-med.ac.jp

Key words: synovial sarcoma, omentum, soft tissue tumor, pazopanib, trabectedin, postmenopause
1 case in a man) have been documented in the literature to date, and they were all associated with an unfavorable clinical course (2-4). Surgical resection and adjuvant chemotherapy involving doxorubicin-ifosfamide are the preferred first-line treatments for synovial sarcoma, whereas pazopanib (a tyrosine kinase inhibitor) and trabectedin (an anticancer alkaloid agent) $(5,6)$ have recently been recommended as treatments for patients with advanced disease, although the optimal therapeutic strategy for primary omental synovial sarcoma has not been clearly determined due to the rarity and severity of the disease.

Large complex abdominal masses that are incidentally discovered in postmenopausal women are often initially suspected to be pelvic malignancies. The lack of characteristic symptoms and specific imaging characteristics may lead to the preoperative misdiagnosis of omental synovial sarcoma as a gynecological malignancy, particularly ovarian cancer. A specific chromosomal aberration, $\mathrm{t}(\mathrm{X} ; 18)(\mathrm{p} 11.2 ; \mathrm{q} 11.2)$, and its product, the SYT-SSX fusion protein, are identified in $>90 \%$ of cases of synovial sarcoma (7), but the fusion gene may only be detected postoperatively based on detailed pathological and immunohistochemical examinations of the tumor.

We herein report a rare case of aggressive primary omental synovial sarcoma mimicking ovarian cancer that was treated with multiple surgical resection procedures and adjuvant chemotherapy involving doxorubicin-ifosfamide, pazopanib and trabectedin.

\section{Case report}

A 51-year-old multigravida woman who experienced abdominal distension for 4 months was referred to the Department of Obstetrics and Gynecology of the Hashimoto Municipal Hospital (Hashimoto, Japan) in April 2014. The patient had no history of lower abdominal or pelvic discomfort, pelvic surgery, or other relevant medical conditions. Transvaginal and transabdominal ultrasound examinations revealed a large solid abdominal mass, which exhibited iso-echogenicity. Magnetic resonance imaging (MRI) revealed a large heterogeneous mass with an irregular component occupying the lower abdominal cavity, with an intact uterus. Based on these radiological findings, the mass was suspected to be an ovarian malignancy (Fig. 1). The results of a laboratory analysis of the patient's peripheral blood revealed normal tumor marker levels [cancer antigen (CA)125, CA19-9 and carcinoembryonic 


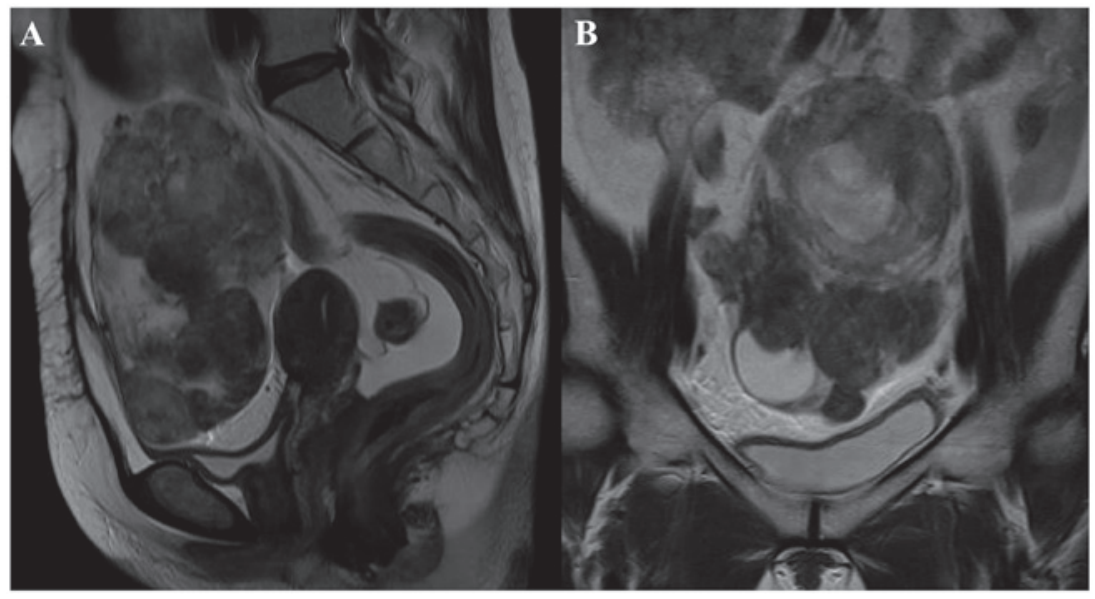

Figure 1. Magnetic resonance imaging of omental synovial sarcoma revealing a large heterogeneous mass with an irregular component. (A) T2-weighted sagittal image (B) T2-weighted coronal image.

antigen] and an elevated lactate dehydrogenase level (291 IU/l). Total abdominal hysterectomy and bilateral salpingo-oophorectomy were planned. Intraoperative examination revealed a solid mass arising from the lower omentum. The resected mass was $12.5 \times 10.0 \times 7.2 \mathrm{~cm}$ in size and weighed $502 \mathrm{~g}$. The consistency of the mass was elastic-hard and partially soft, and its cut surface was whitish-yellow and contained a cystic and necrotic component measuring $2 \mathrm{~cm}$ in diameter. Microscopically, the tumor included a well-circumscribed region composed of hyaline-fibrous and mucinous-myxomatous components. The hyaline-fibrous region exhibited diffuse proliferation of spindle-shaped and small round tumor cells, with capillaries arranged in a spider's web-like pattern (Fig. 2). The mucinousmyxomatous region was composed of highly cellular tissue, which displayed a high nuclear:cytoplasmic ratio and mitotic figures. Immunohistochemistry revealed positive staining of the tumor cells for B-cell lymphoma (Bcl)-2, and partially positive staining for CD99 and epithelial membrane antigen (EMA). The tumor cells were negative for S100, $\alpha$-smooth muscle actin (SMA), desmin, CD34, CD31, cytokeratin (CK)7 and CK AE1/3. The Ki-67 labeling index was 40-60\%. Immunohistochemistry helped to exclude leiomyosarcoma, malignant schwannoma, gastrointestinal stromal tumor and solitary fibrous tumor. Although a qualitative analysis of mRNA expression based on a reverse transcription polymerase chain reaction (RT-PCR) assay was performed using an RNA sample extracted from formalinfixed paraffin-embedded tumor tissue, the SYT-SSX fusion gene transcript was not detected. The diagnosis of primary omental poorly differentiated synovial sarcoma was confirmed based on the pathological findings. The patient's postoperative course was uneventful, and she was discharged from the hospital on postoperative day 7 . The patient was treated with a combination of doxorubicin and ifosfamide as adjuvant chemotherapy. Despite normal findings on physical examination, 7 months after the operation a metastatic tumor was detected in the liver by positron emission tomography-computed tomography (PET-CT), and laparoscopic partial liver resection was performed, followed by adjuvant pazopanib. Twelve months after the operation, another metastatic tumor was detected in the liver by PET-CT, and the patient was again treated with laparoscopic partial liver resection followed by adjuvant pazopanib. At 14 months after

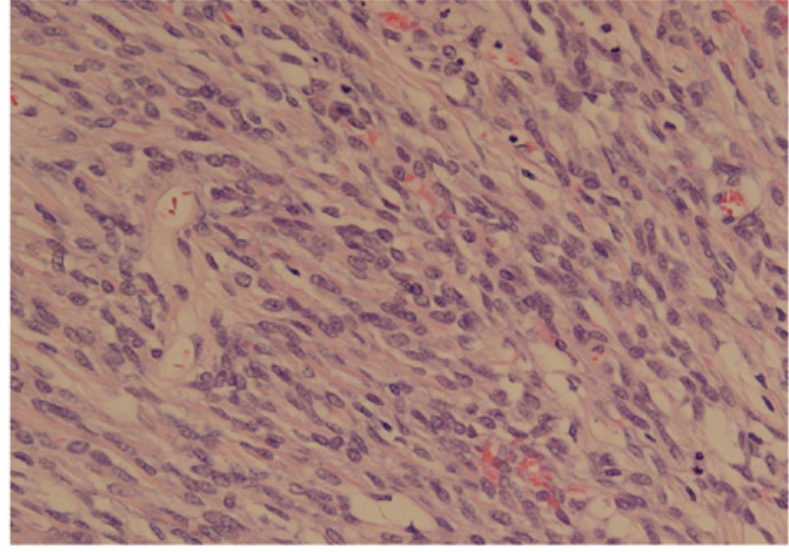

Figure 2. Hematoxylin and eosin staining of omental synovial sarcoma, showing diffuse proliferation of spindle-shaped and small round tumor cells (magnification, x100).

the first operation, PET-CT revealed multiple recurrent metastatic tumors in the liver, lungs and pleura. Although the patient was treated with partial lung resection, aggressive metastatic tumors were detected in the liver, lungs and abdominal cavity at 2 months after the first operation. Due to the difficulty of surgical resection, the patient was treated with trabectedin, and she remained alive with stable disease at 24 months after the first operation. The patient provided written informed consent regarding the publication of the case details and associated images.

\section{Discussion}

Synovial sarcoma is a rare soft tissue malignant tumor. Despite its name, synovial sarcoma does not necessarily arise from synovial or soft tissue and has been reported to develop at other sites, such as the kidneys, lungs and pleura. Synovial sarcoma most commonly develops in the extremities, particularly in the knee, but primary intra-abdominal synovial sarcoma has rarely been reported, with only 8 cases published in the literature to date (2).

Only 3 cases of primary omental synovial sarcoma have been reported in the English literature since 1945, according to 
a search through the PubMed and MEDLINE databases (2-4): 2 of the cases involved women, aged 16 and 37 years $(2,3)$, and the remaining case involved a man, aged 66 years (4). Systemic symptoms, such as abdominal pain, fullness, tenesmus and vomiting, occurred in these cases, and the tumors ranged in size from 9.5 to $20 \mathrm{~cm}$. None of these cases were diagnosed as omental synovial sarcoma preoperatively based on radiological examinations; instead, they were diagnosed postoperatively based on detailed pathological and immunohistochemical examinations of the tumor.

The diagnosis of primary omental synovial sarcoma is difficult in female patients, as surgery is required for a definitive diagnosis. In a previous study, the results of imaging analysis were non-specific (8): On MRI, T1-weighted imaging (WI) revealed areas of isointensity or hyperintensity relative to muscle in the tumor masses, and T2WI frequently showed heterogeneous signal intensity, including areas of triple signal intensity involving regions of high signal intensity that appeared fluid, isointense, or hyperintense relative to fat, and hypointense relative to fibrous tissue. In a case involving a postmenopausal female, a malignant tumor (ovarian cancer) was initially suspected due to the detection of a large abdominal mass and signs of necrosis.

Histologically, poorly differentiated synovial sarcoma is formed from sheets of undifferentiated round cells with hyperchromatic nuclei, which exhibit frequent mitoses, whereas biphasic synovial sarcoma is composed of a combination of epithelial and spindle cell components, and monophasic synovial sarcoma is diagnosed when only an epithelial or a spindle cell component is observed (1). Immunohistochemical studies may confirm the pathological diagnosis. Poorly differentiated synovial sarcoma is usually positive for Bcl-2 and CD99, and focally positive for EMA, but it is negative for S-100, $\alpha$-SMA, desmin, CD34 and CD31. Poorly differentiated synovial sarcoma differs from biphasic or monophasic synovial sarcoma in that it is also negative for CK7 and CK AE1/3, which emphasizes its poorly differentiated status and the nonepithelial nature of the tumor cells. The SYT-SSX fusion gene, which is produced by the chromosomal translocation $\mathrm{t}(\mathrm{X} ; 18)$ (p11.2;q11.2), is found in $\sim 90 \%$ of synovial sarcomas during fluorescence in situ hybridization or RT-PCR analysis of the gene transcript (7). Although previous studies have detected the SYT-SSX fusion gene in omental synovial sarcoma $(2,3)$, its true frequency in omental poorly differentiated synovial sarcoma remains unknown. Even if molecular testing for the SYT-SSX fusion gene produces a negative result, the diagnosis of synovial sarcoma may be confirmed based on immunohistochemical findings. While the product of the SYT-SSX fusion gene was not detected in the present case, immunohistochemistry detected positivity for Bcl-2, focal positivity for CD99 and EMA, and negativity for S100, $\alpha$-SMA, desmin, CD34, CD31, CK7 and CK AE1/3. Thus, a final diagnosis of poorly differentiated omental synovial sarcoma was made.

The most appropriate clinical management strategy for primary omental synovial sarcoma may not be clear, as our experience with such cases is limited. According to the 3 previous reports on this tumor (2-4), omental synovial sarcoma appears to have a poor prognosis, although synovial sarcomas characteristically progress slowly. The primary treatment for this disease is surgery, but in all 3 reported cases of omental synovial sarcoma, multiple aggressive metastases developed following complete resection. Despite receiving combined treatment with surgery and chemotherapy, the patients succumbed to the disease within 2 months, 13 months and 3 years, respectively. Patients who present with local recurrence, distant metastasis, incomplete resection, positive margins, lymph node involvement, or large bulky tumors, should undergo adjuvant chemotherapy involving doxorubicin-ifosfamide $(5,6)$. Some molecular-targeted drugs, including pazopanib, a selective multi-targeted receptor tyrosine kinase inhibitor, and trabectedin, an anticancer alkaloid agent, have been recommended as second-line chemotherapies in cases of poorly differentiated synovial sarcoma in which combined treatment with doxorubicin and ifosfamide fails to control metastasis, as they block tumor growth and inhibit angiogenesis $(5,6)$. In the present case, the tumor progressed rapidly, with multiple metastases and locally recurrent lesions developing during the short follow-up period, which were treated with aggressive surgical resection and adjuvant chemotherapy. The rapid tumor progression observed in this case was consistent with the previous 3 reports. Although treatment with pazopanib and trabectedin was well-tolerated, it was ultimately unsuccessful. To improve the treatment of omental synovial sarcoma, further data regarding its natural history, diagnosis and treatment are required.

In summary, we encountered a rare case of primary omental synovial sarcoma, in which aggressive metastasis developed and proved difficult to control with a combination of surgical and chemotherapeutic interventions, including molecular-targeted drugs. Gynecologists should be aware of the aggressive metastatic potential of this rare tumor and the importance of an intensive diagnostic approach. Furthermore, a new therapeutic strategy for primary omental synovial sarcoma is required to effectively control this disease.

\section{References}

1. Fisher C, de Bruijn DRH and Geurts van Kesse A: Synovial sarcoma. In: WHO pathology and genetics: tumours of soft tissue and bone. Fletcher CDM, Unni KK and Mertens F (eds). IARC Press, Lyon, France, pp200-204, 2002.

2. Indranil G and Divya M: Synovial sarcoma of the omentum: A rare entity. Indian J Cancer 52: 166-167, 2015.

3. Hemmings $C$ and Fisher C: Primary omental synovial sarcoma: A case with cytogenetic confirmation. Pathology 36: 208-211, 2004.

4. Travaglini G, Biagetti S, Alfonsi S, Bearzi I and Marmorale C: Primary intra-abdominal synovial sarcoma: A case report. Ann Ital Chir: Sep 3, 2013 (Epub ahead of print).

5. van der Graaf WT, Blay JY, Chawla SP, Kim DW, Bui-Nguyen B, Casali PG, Schöffski P, Aglietta M, Staddon AP, Beppu Y, et al; EORTC Soft Tissue and Bone Sarcoma Group; PALETTE study group: Pazopanib for metastatic soft-tissue sarcoma (PALETTE): A randomised, double-blind, placebo-controlled phase 3 trial. Lancet 379: 1879-1886, 2012.

6. Sanfilippo R, Dileo P, Blay JY, Constantinidou A, Le Cesne A, Benson C, Vizzini L, Contu M, Baldi GG, Dei Tos AP, et al: Trabectedin in advanced synovial sarcomas: A multicenter retrospective study from four European institutions and the Italian Rare Cancer Network. Anticancer Drugs 26: 678-681, 2015.

7. Aubry MC, Bridge JA, Wickert R and Tazelaar HD: Primary monophasic synovial sarcoma of the pleura: Five cases confirmed by the presence of SYT-SSX fusion transcript. Am J Surg Pathol 25: 776-781, 2001.

8. Liang C, Mao H, Tan J, Ji Y, Sun F, Dou W, Wang H, Wang H and Gao J: Synovial sarcoma: Magnetic resonance and computed tomography imaging features and differential diagnostic considerations. Oncol Lett 9: 661-666, 2015. 